\title{
Contributions to Zoology thanks the following reviewers
} for their assistance over the 2015 calendar year:

Ainara Achurra
Jason Anderson
Hannes Baur
Martin Brazeau
Brian Brown
Niels Bruce
Alfredo Castro Vazquez
Todd Cook
Regina Cunha
Matthieu Denoël
Henry Disney
Tom van Dooren
Abby Drake
Zdenek Duris

\author{
Sarah Elton \\ Renata Freitas \\ Frank Friedrich \\ Viviana Frisone \\ Aida Gomez-Robles \\ Wouter Halfwerk \\ Joachim Haug \\ Ken Hayes \\ Kentaro Inoue \\ Herman de Jong \\ Pepijn Kamminga \\ Jürgen Kriwet \\ Martjan Lammertink \\ Kara Layton
}

Malgorzata Lesniewska
Iain McGaw
Jimmy McGuire
Marcello Mezzasalma
Thibaut de Meulemeester
Diane Nelson
David Obura
Gaetano Odierna
Marco Oliverio
Mateus Pepinelli
Giovanni Pilato
Alexey Polilov
Alex Pyron
Menno Reemer

Dominique Roche

Nadia Santodomingo

Koh Siang Tan

Eduard Sola

Paolo Sordino

Jarosław Stolarski

Randy Thornhill

Tarmo Timm

Ben Titus

Varpu Vahtera

Maarten Vanhove

Miquel Vences

Alexandra Zieritz

In 2015 Contributions to Zoology was printed on 24 March (84-1), 31 August (84-2), 16 October (84-3) and 28 December (84-4) 